\title{
Estudio de los sistemas silvopastoriles como alternativa para el manejo sostenible de la ganadería
}

\section{Study of silvopastoral systems as an alternative for the sustainable management of livestock}

Fecha de recepción: 4 de abril de 2018

Fecha de aprobación: 18 de junio de 2018
Sandra Patricia Arciniegas-Torres ${ }^{1}$

Dixon Fabián Flórez-Delgado ${ }^{2}$

DOI: http://doi.org/10.19053/01228420.v15.n2.2018.8687

\section{Resumen}

Los sistemas silvopastoriles son vistos como una solución factible para la ganadería con enfoque de sostenibilidad. Se destaca que la ganadería contribuye con la seguridad alimentaria, al generar productos alimenticios, empleos e ingresos; sin embargo, también ha generado impactos negativos en el ambiente, como lo son la compactación del suelo, el detrimento de la biodiversidad, la deforestación, la contaminación de fuentes hídricas y la emisión de gases de efecto invernadero, que contribuyen al cambio climático, uno de los inconvenientes ambientales más preocupantes, ya que perjudica a todos los seres vivos del planeta. Por esta razón, se hace indispensable ahondar en el tema de los sistemas silvopastoriles, sus tipos, ventajas y desventajas, con el fin de presentarlos como una alternativa de solución que contribuye con la mitigación del efecto que tiene la ganadería sobre el medioambiente, representando una opción viable para su conservación y adaptación al cambio climático al permitir intensificar la producción ganadera mediante la optimización y uso racional de los recursos naturales.

Palabras clave: abastecimiento alimenticio; ecología animal; producción alimentaria; producción bovina.

\begin{abstract}
Silvopastoral systems are seen as a feasible solution for livestock with a focus on sustainability, particularly because they contribute to food security, by generating food products, jobs and income. However, livestock has also generated negative impacts on the environment, such as soil compaction, detriment of biodiversity, deforestation, pollution of water sources and emission of greenhouse gases, which contribute to climate change, this being one of the most worrisome environmental problems, since it harms all

1 Universidad Nacional Abierta y a Distancia - UNAD (Bucaramanga-Santander, Colombia).

2 M. Sc. Universidad de Pamplona (Pamplona-Norte de Santander, Colombia).dixon.florez@unipamplona.edu.co. ORCID: 0000-0002-3915-8396.
\end{abstract}

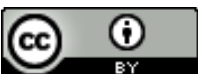


living beings on the planet. For this reason, it is essential to delve into the issue of silvopastoral systems, their types, advantages and disadvantages to present them as an alternative solution that contributes to mitigate the livestock effect on the environment, representing a viable option for its conservation and adaptation to climate change; this is achieved by allowing the intensification of livestock production through the optimization and rational use of natural resources.

Keywords: animal ecology; cattle production; food production; food supply.

\section{Para citar este artículo:}

Arciniegas-Torres S.P., Flórez-Delgado D.F. Estudio de los sistemas silvopastoriles como alternativa para el manejo sostenible de la ganadería. Ciencia y Agricultura. 2018; 15(2): 107-116. 


\section{Introducción}

La importancia de la ganadería radica, especialmente, en su contribución a la seguridad alimentaria, debido a los diferentes productos que proporciona, como la carne, que contiene niveles significativos de vitaminas, proteínas, micronutrientes y minerales, que son fundamentales para el proceso de desarrollo de las personas (1), y la leche, que se destaca por ser un alimento considerado de primera necesidad y por presentar una gran demanda, gracias al alto valor nutricional que tiene (2). La ganadería, además, contribuye de manera significativa con la generación de ingresos y empleos; según datos de FEDEGAN, esta actividad ofrece aproximadamente 950.000 empleos, representando una participación del $7 \%$ del empleo nacional (3). Pero la ganadería es una de las labores que ha generado más efectos negativos en el medioambiente (4), por ello, este trabajo se basa en el análisis de los sistemas silvopastoriles (5), como una alternativa viable para tener en cuenta en el momento de establecer un sistema productivo ganadero que sea rentable y que permita llevar a cabo un manejo sostenible, en asocio con la conservación del medioambiente (6).

\section{Sistemas de producción ganaderos en la seguridad alimentaria}

La ganadería se conoce como una actividad que se enfoca en la cría de bovinos, con el fin de utilizar los productos que pueden obtenerse de estos animales (7); se destaca la gran importancia que tiene, debido a la diversidad de productos y servicios que proporcionan los bovinos, ya que contribuye significativamente con la seguridad alimentaria, principalmente en los países de bajos ingresos y carencia de alimentos; además, es fundamental tener presente que, según la $\mathrm{FAO}$, la producción ganadera contribuye con alrededor del $40 \%$ de la producción agrícola en el mundo, y es considerada el soporte de las vías de manutención y la disponibilidad de alimentos para alrededor de mil millones de habitantes, siendo las actividades ganaderas las que aportan el $15 \%$ del total de la energía alimentaria, así como también contribuyen con el $25 \%$ de las proteínas incorporadas en la dieta, teniendo en cuenta que la leche y la carne son productos que proveen micronutrientes esenciales que no se adquieren buenamente de otros alimentos de origen vegetal (8).

\section{A. Producción mundial de carne de bovino}

Entre los años 2007 y 2016, según FIRA (Fideicomisos Instituidos en Relación con la Agricultura), se presentó un notable crecimiento de la producción, con un promedio anual de $0.3 \%$; aunque es de mencionar que se presentaron tasas de decrecimiento en algunos países productores importantes, como Estados Unidos, cuya producción disminuyó a una tasa promedio por año de $0.6 \%$; en Unión Europea, un $0.5 \%$; en Argentina, un $2.2 \%$, y en Australia, un $0.2 \%$. Sin embargo, cabe destacar que en dicho período la producción creció en Turquía a razón de $14.3 \%$ promedio anual; en India, un $6.2 \%$; en Paquistán, un $3.0 \%$; en México, un $1.8 \%$, y en China, un $1.3 \%$. Al observar la figura 1 se evidencia que en el año 2016 la producción mundial de carne de bovino fue de 60.5 millones de toneladas (9) y en el año 2017 ascendió a una nivelación récord de 61.3 millones de toneladas, presentando un incremento por año de $1.4 \%$ (10). 


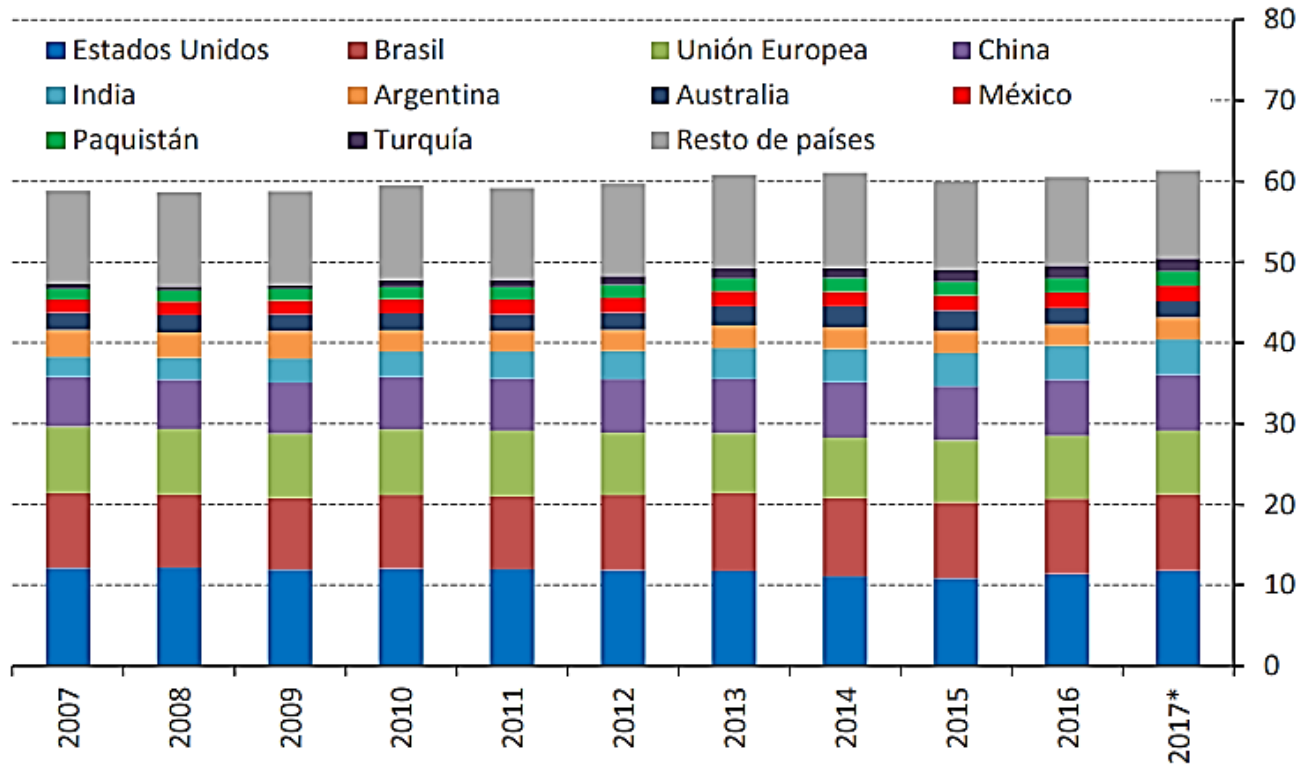

Fig. 1. Producción mundial de carne de bovino, 2007-2017 (Millones de toneladas, equivalente en canal) (11).

\section{B. Consumo mundial de carne de bovino}

Los países que más se destacan por consumir carne de bovino son Estados Unidos, Brasil, Unión Europea, China, Argentina, Rusia, India, México y Pakistán, que alcanzaron su nivel máximo en 2007, cuando registraron un consumo de 45.2 millones de toneladas; sin embargo, es importante dar a conocer que entre los años 2007 y 2014 el consumo en estos países presentó altas y bajas, pues durante el año 2014 el consumo fue de 44.2 millones de toneladas, por lo cual, para el 2015, se esperaba un ligero aumento en el consumo de un 0.04 por ciento anual, esto teniendo en cuenta que, el consumo mundial de carne bovino para el año 2013 fue de 56 millones de toneladas, ubicándose un $1 \%$ por debajo en el año 2012 (12). Por otra parte, se pudo evidenciar que el país que es considerado el mayor consumidor de carne de bovino es Uruguay, con un consumo estimado de 59,4 kg per cápita, para el año 2017, lo que representa un incremento de $1,6 \mathrm{~kg}$ con relación al consumo presentado en el año 2016 (13). Además, es importante dar a conocer que la OCDE-FAO han realizado unas proyecciones sobre el consumo mundial de carne de bovino, por lo cual se espera que dicho consumo avance de nuevo en la próxima década, aunque es de tener en cuenta que aumentará solo $0,1 \mathrm{~kg}$ por persona en 2023, al verificar con el promedio de la etapa base (14).

\section{Impacto ambiental de la ganadería}

Según la FAO, la producción ganadera es considerada una de las mayores fuentes de los inconvenientes ambientales más preocupantes en el mundo, dentro de los cuales se encuentra el aumento de la temperatura terrestre, la afectación de tierras, el detrimento de la biodiversidad, las afectaciones a la atmósfera y la contaminación de fuentes hídricas; se afirma que la ganadería bovina genera el $18 \%$ de los gases que incrementan el efecto invernadero (15), teniendo en cuenta que produce dióxido de carbono $\left(\mathrm{CO}_{2}\right)$ a razón de un $27 \%$; metano $\left(\mathrm{CH}_{4}\right)$, en una proporción de $44 \%$, y óxido nitroso $\left(\mathrm{N}_{2} \mathrm{O}\right)$, a razón de $29 \%$ (16). En cuanto al cambio climático, se hace referencia a las labores desarrolladas por las personas, las cuales generan alteraciones en la estructura de la atmósfera (17); dentro de los cambios que trae consigo se encuentra el incremento de la temperatura, de las precipitaciones y del nivel del mar; debido a estas modificaciones, se presenta una notable reducción de la disponibilidad de agua (18), viéndose afectada la 
cantidad de agua para suministrarles a los bovinos; así también, se han dado a conocer impactos negativos a nivel nutricional y sanitario (19), teniendo en cuenta que se alteran las características de los alimentos que van a consumir los animales, se modifican las exigencias tanto de agua como de energía y, a su vez, se altera el uso que los bovinos le dan; a su vez, se incrementa la presencia de plagas, generando cambios en el consumo, en la conducta y en los índices productivos y reproductivos de los bovinos (20).

\section{Sistemas silvopastoriles}

Los sistemas silvopastoriles son agroecosistemas en los que se asocia a propósito, en un mismo sitio, un componente arbóreo (árboles, arbustos, palmas y otros) con uno herbáceo (cultivos, pasturas) y un componente pecuario como el ganado, principalmente, buscando interacciones biológicas entre estos componentes, con el objetivo de maximizar el uso que se le brinda a la tierra (21). Al desarrollar una mezcla de arbustos forrajeros, árboles y pastos con la producción bovina, los sistemas silvopastoriles representan una buena alternativa para contribuir con la mitigación del efecto que ocasiona la actividad ganadera sobre el medioambiente (22), debido a que estos sistemas se caracterizan por ayudar a conservar la cubierta vegetal, aportando fertilidad con el paso del tiempo, así como también por contribuir con la producción animal, al generar rentabilidad (23); además de destacar que el silvopastoreo es considerado un método efectivo para mitigar los efectos del cambio climático (24).

\section{A. Principales tipos de sistemas silvopastoriles}

Estos representan las distintas combinaciones o formas de integrar plantas leñosas perennes con pasturas herbáceas y animales, destacando que en la ganadería se busca implementar los sistemas silvopastoriles con el fin de facilitar la alimentación del ganado, pues al diseñarlos de manera adecuada pueden estabilizar la provisión de alimento para los animales, manteniendo la diversidad de forrajes y pasturas, generando una utilidad a nivel ecológico, económico y social; por lo cual es fundamental dar a conocer que entre las alternativas silvopastoriles que pueden ponerse en funcionamiento en una producción ganadera se encuentran las cercas vivas, los bancos mixtos de forraje, las pasturas en callejones y el establecimiento de árboles frutales o maderables en las praderas (25).

\section{B. Ventajas de los sistemas silvopastoriles}

Los sistemas silvopastoriles permiten obtener múltiples beneficios, dentro de los cuales se destacan los siguientes:

- Mejorar la fertilidad del suelo. La integración de arbustos y árboles (leñosas perennes) en la producción ganadera ayuda a enriquecer la estructura del suelo (26). Implementar el uso de gramíneas junto con los árboles o arbustos posibilita que parte considerable de nutrientes que son extraídos del suelo sean devueltos a este por medio de la defecación de los animales sobre el suelo y el follaje, y mediante los residuos de pastoreo; se destaca que gran cantidad de la evacuación de la materia orgánica ayuda a reformar las propiedades físicas que presenta el suelo (27), pudiéndose ver alterada la estructura. Para la fijación de nitrógeno es muy beneficioso implementar leguminosas, ya que estas se asocian con unas bacterias Ilamadas Rhizobium, que captan nitrógeno atmosférico y lo hacen disponible para las gramíneas en el suelo; se destaca que dicho nitrógeno beneficiará a la planta que se asocie con estas bacterias $y$, finalmente, el beneficio se esparce a los pastos o cultivos cercanos; además de que posibilita el hecho de sustituir los abonos nitrogenados y minimizar el valor de la fertilización (28).

- Mejorar la actividad de la microfauna y la macrofauna. Gracias a que el suelo contiene materia orgánica y a las condiciones climáticas generadas por los árboles, la acción biológica de la fauna se ve favorecida, trayendo como consecuencia un incremento en la mineralización y el nitrógeno disponible en el suelo. Asimismo, cabe mencionar que la incorporación de materia orgánica al suelo se da de manera progresiva mediante la actividad de la endo- 
fauna (29) y ayuda a renovar la estabilidad del suelo y la disposición de infiltración del agua.

- Disminuir los procesos de erosión. Los árboles que se encuentran en los sistemas silvopastoriles ejecutan unas funciones ecológicas respecto al cuidado del suelo, las cuales ayudan a disminuir el impacto directo que proviene del sol, de los vientos y del agua (30), contribuyendo al control de la erosión.

- Regular el estrés climático de los animales. La implementación de plantas leñosas perennes en la ganadería contribuye directamente a la productividad del sistema, debido a que ayuda a regular o contrarrestar la potencia de los factores adversos del clima sobre el animal, permite un mayor consumo de alimentos por los animales, los cuales dedican más tiempo a pastorear y rumiar, mejorando los parámetros productivos y reproductivos, y, a su vez, contribuye de manera indirecta al generar condiciones climáticas que benefician la calidad (31) y el proceso de desarrollo de las pasturas.

\section{Desventajas de los sistemas silvopastoriles}

Los sistemas silvopastoriles pueden generar algunas desventajas, como lo son:

- La competencia por la luz, ya que la sombra generada por los árboles en estos sistemas puede afectar el rendimiento de algunas especies de gramíneas y, a su vez, se ve afectado el rendimiento total del sistema agropecuario (32) en caso de que las especies arbóreas no sean consumidas por los bovinos.

- La presencia de plagas. Implementar plantas forrajeras o herbáceas asociadas en un sistema silvopastoril puede Ilamar la atención de vectores de enfermedades o plagas (33) que perjudican las plantas. La alelopatía, que hace referencia a una interrupción química que se da de las pasturas a las leñosas o viceversa, manifestando una acción de dominancia diferencial y exclusión que presentan algunas plantas (34), puede afectar el proceso de desarrollo y la supervivencia de otras especies de plantas.
- El ramoneo. El tránsito libre de los animales en las zonas de los silvopastoriles donde se encuentran las leñosas ocasiona daños a estas (35), razón por la cual es de gran importancia tener en cuenta el manejo y, a su vez, es indispensable proteger las plantas de un ramoneo precoz de los animales mientras estas se establecen.

\section{Aportes de los sistemas silvopastoriles al ambiente}

Los sistemas silvopastoriles se han dado a conocer por desarrollarse mediante el uso de tecnologías que involucran el progreso de los sistemas ganaderos al mismo tiempo que proporcionan beneficios ambientales por medio de técnicas que se emplean en las diferentes actividades agrícolas en las que se aprecian los árboles y los arbustos como componentes esenciales para la industria animal, teniendo en cuenta que estos ayudan a aumentar la calidad y el rendimiento de los pastos, contribuyen con la reducción de la emanación de gases efecto invernadero $y$, a su vez, interceden en la conservación de la biodiversidad, al amparar las cuencas hídricas y enriquecer las propiedades biológicas y fisicoquímicas del suelo (36).

\section{A. Fijación biológica de nitrógeno}

Conocida como FBN, por su sigla, hace referencia a la reducción de nitrógeno a amonio, la cual es efectuada por bacterias de vida libre o en simbiosis con algunas especies vegetales, entre las cuales es posible mencionar las leguminosas y algunas leñosas no leguminosas, representando así una posibilidad de fertilización nitrogenada debido a que puede mitigar algunos de los impactos perjudiciales a nivel sanitario y ambiental que son ocasionados por dicha fertilización. Además, es de destacar que la FBN es relegada a seres procariotas, que ayudan a sintetizar el nitrógeno molecular a amoniaco, ya sea por simbiosis o en vida libre, destacando que en dicha fijación una considerable cantidad del nitrógeno es fijado en los ecosistemas terrestres, mediante la alianza simbiótica de bacterias de los géneros Bradyrhizobium, Rhizobium, Sinorhizobium, Mesorhizobium y Azorhizobium 
(Rhizobium para generalizar) con plantas leguminosas (37).

\section{B. Producción de madera y frutos}

Los árboles que se encuentran establecidos de manera dispersa en las praderas son una opción viable para llevar a cabo una producción de madera sostenible, gracias al manejo silvicultural de la regeneración natural (38); en cuanto a la implementación de árboles frutales en los sistemas silvopastoriles es posible indicar que es recomendable, debido a que estos cuentan con muy buenas características para ser empleados en la alimentación del ganado, ya sea como fuente de forraje, como alimento energético, proteína, vitaminas y minerales (39).

\section{Captación de dióxido de carbono}

Para analizar la captación de este gas de efecto invernadero se debe, inicialmente, tener claro que el CO2 es considerado el compuesto más importante en lo que refiere al tema del calentamiento global, esto teniendo en cuenta el volumen de este gas que se produce al año, el tiempo que se queda en la atmósfera y el incremento en su concentración atmosférica, absorbiendo la radiación térmica y provocando alrededor del $50 \%$ del calentamiento global (40); por lo tanto, se han planteado los sistemas silvopastoriles como una forma de desarrollar la producción pecuaria integrando leñosas perennes, forrajeras herbáceas y animales, con el objetivo de aumentar la rentabilidad del sistema; $y$ es que, aunque anteriormente no se menciona, los sistemas silvopastoriles también incluyen el componente suelo, de manera que necesariamente se presentarán relaciones entre el suelo, las leñosas, las herbáceas y los animales, destacando que cada uno de dichos componentes pueden representar una fuente de carbono o un sumidero (41).

\section{Liberación de oxígeno}

El proceso de liberación de oxígeno se da, principalmente, mediante la fotosíntesis de las plantas, proceso mediante el cual estas producen energía química a partir de la energía lumínica que reciben del sol, gracias a la cual las plantas pueden conver- tir el agua, que obtienen del suelo, y el dióxido de carbono, que reciben del aire, en glucosa, que es una fuente de energía para estas (42), y a su vez en este proceso se libera oxígeno como producto sobrante.

\section{E. Servicios en la preservación de la biodiversidad}

Es bien sabido que la transformación de los bosques en pasturas está amenazando la conservación de la vida de muchas especies; sin embargo, es de destacar que el efecto que genera sobre la biodiversidad en los bosques puede ser reducido, en caso de que los productores implementaran especies arbóreas y forestales en las praderas, teniendo en cuenta que dichos árboles servirían como corredores biológicos para proporcionar alimento y hábitat, lugares de descanso y refugio para fauna silvestre y avifauna nativa y migratoria, y a su vez serán productores de semillas (43). Además, de destacar que la inclusión de árboles en las pasturas también puede generar condiciones propicias por medio del suministro de materia orgánica, la disminución de la temperatura, la humedad presente en el suelo y el ciclaje de nutrientes (44), además de que estos sistemas permitirán establecer una conexión en forma de corredor el cual tiene gran influencia sobre la difusión de las plantas y el desplazamiento de los bovinos (45).

\section{F. Secuestro de carbono}

El secuestro de carbono se da en este tipo de sistemas debido a que estos añaden dos utilidades significativas para preservar carbono (46); la primera utilidad es el hecho de contribuir al acopio directo de carbono a mediano y corto plazo, y la segunda se da al ayudar a reducir, de manera indirecta, la emanación de gases de efecto invernadero como resultado de las actividades pecuarias, agrícolas y de deforestación; y es que es de tener presente que si se le brinda un adecuado manejo a los sistemas silvopastoriles, estos muy posiblemente mejorarán la productividad del sistema productivo (47), mientras secuestran carbono. Por otra parte, es fundamental aclarar que la cantidad de carbono que es sujetada en los sistemas silvopastoriles puede verse perjudicada por la especie de leñosas y forrajeras que se implementan, por la densidad 
de la distribución de las leñosas en la pradera, así como por la tolerancia y resistencia de estas especies a la sombra (48), aspectos que se deben analizar al momento de querer instaurar un sistema silvopastoril y buscar obtener los beneficios que estos proporcionan.

\section{Resultados y discusión}

Al analizar los impactos ambientales de la actividad ganadera en el mundo, se pudo constatar que genera problemas ambientales y que los bovinos son causantes de una cantidad significativa de emanaciones de gases que generan el efecto invernadero; gases que son producidos a lo largo de todo el proceso productivo ganadero, a partir de la trasformación de los bosques en terrenos para pasturas, durante el proceso de digestión de los bovinos y a causa de las flatulencias de dichos animales, situación que ha generado una serie impactos ambientales, entre los que sobresalen el calentamiento global, la degeneración del suelo, afectaciones a las fuentes hídricas, al aire y a la biodiversidad (49), que son hoy por hoy temas muy preocupantes en el mundo.

El cambio climático también afecta el sector ganadero, ya que, al provocar un incremento de la temperatura, al generar alteraciones y disminuciones de las Iluvias y al alargar la temporada seca, ocasiona problemas preocupantes en la ganadería en lo que respecta a su productividad y rendimiento, al dificultarse el acceso al agua de los animales, al presentarse una reducción en el valor nutricional de los forrajes, así como en su valor nutritivo al presentarse estrés calórico en los bovinos a causa de las altas temperaturas, y también a causa de la presencia de vectores y plagas. Teniendo en cuenta los impactos negativos que ocasiona la ganadería, se hace necesario buscar una alternativa que permita un manejo sustentable de dicha actividad; sobresaliendo este tipo de sistemas de producción como una alternativa viable para tal fin, la cual permite intensificar la productividad del sistema por medio de la adecuada utilización de los recursos naturales, contribuyendo con la protección del medioambiente (50).

\section{Conclusiones}

Los sistemas silvopastoriles se dan a conocer como una estrategia viable, mediante la cual los productores pueden intensificar la producción ganadera brindando un manejo apropiado de los recursos naturales, lo cual ayudaría a reducir los efectos ambientales de las actividades como la ganadería, permitiéndole al productor mejorar la rentabilidad del sistema, a la vez que propugna el cuidado del ambiente.

Los sistemas silvopastoriles contribuyen con la disminución de los gases que generan el efecto invernadero y que se relacionan directamente con el cambio climático, y ayudan a diversificar la producción, mejorando su rentabilidad mediante el uso racional de los recursos naturales, al propiciar una producción sustentable a nivel técnico, productivo y económico.

La ganadería representa un pilar fundamental para la seguridad alimentaria, dado que brinda carne y leche, que son importantes para un adecuado desarrollo de las personas, teniendo en cuenta que aportan valiosos nutrientes, por lo cual es indispensable propiciar su desarrollo, de manera sustentable, rentable y que no afecte negativamente el ambiente.

\section{Referencias}

(1) Organización de las Naciones Unidas para la Alimentación y la Agricultura FAO. Consumo de Carne. 2014. Disponible en: https://goo.gl/owY7At.

(2) Agudelo Gómez D.A., Bedoya Mejía O., Composición nutricional de la leche de ganado vacuno. Rev. Lasallista Investig. 2005; 2(1): 38-42.

(3) Federación Colombiana de Ganaderos FEDEGAN. Plan estratégico de la ganadería colombiana 2019. 2016. Disponible en: https://goo.gl/UcmePC

(4) Pérez Espejo R. Testimonio: El Lado Oscuro de la Ganadería. Problemas Del Desarrollo. Rev. Latinoamericana de Economia. 2008, 39(154): 217-227.

(5) Russo R.O. Reflexiones sobre los sistemas silvopastoriles. Pastos y Forrajes. 2015; 38(2): 157-161.

(6) López Vigoa O., Sánchez Santana T., Iglesias Gómez J.M., Lamela López L., Soca Pérez M., Arece García J., Milera Rodríguez M.C. Los sistemas silvopastoriles como alternativa para la producción animal sostenible en el contexto actual de la ganadería tropical. Pastos y Forrajes. 2017; 40(2): 83-95. 
(7) Carmona Carmona M.H., González Pulgarín D.E. Una Propuesta de Sistema de Costos para el Sector Ganadero. Trabajo de grado, Facultad de Contaduria Publíca, Universidad de Antioquia, 2010.

(8) Organización de las Naciones Unidas para la Alimentación y la Agricultura FAO. Hacia un sector ganadero más sostenible. 2010. Disponible en: https://goo.gl/Cni5Uf

(9) Fideicomisos Instituidos en Relación con la Agricultura FIRA. Panorama Agroalimentario, Carne Bovino 2017. Dirección de Investigación y Evaluación Económica y Sectorial; 2017. Disponible en: https://goo.gl/YBY1d5

(10) Blasina y Asociados. La producción mundial de carne vacuna de 2017 será récord. 2016. Disponible en: https://goo.gl/51nE8B

(11) Fideicomisos Instituidos en Relación con la Agricultura FIRA. Panorama Agroalimentario, Carne Bovino 2015. Dirección de Investigación y Evaluación Económica y Sectorial; 2015. Disponible en: https://goo.gl/0SrS4u

(12) Errecart V., Lucero M,, Sosa M.A. Análisis del mercado mundial de carnes. Facultad de Economía y Negocios, Universidad Nacional de San Martín, 2015.

(13) Instituto Nacional de Carnes INAC. Evaluación del año en INAC. 2017. Disponible en: https://goo.gl/nBy4VE

(14) Boari R., Chuard N., Fernández V., Pouiller P. Mercado de Ganados y Carnes Proyecciones 2023. Organización para la Cooperación y el Desarrollo Económicos - Organización de las Naciones Unidas para la Alimentación y la Agricultura OCDE - FAO 2014. Disponible en: https://goo.gl/NGujiZ

(15) Organización de las Naciones Unidas para la Alimentación y la Agricultura FAO. Las repercusiones del ganado en el medio ambiente. Departamento de Agricultura y Protección del Consumidor 2006. Disponible en: https://goo.gl/AcqhQ

(16) Gerber P.J., Steinfeld H., Henderson B., Mottet A., Opio C., Dijkman J., Falcucci A., Tempio G. Enfrentando el cambio climático a través de la ganadería-Una evaluación global de las emisiones y oportunidades de mitigación. Organización de las naciones unidas para la alimentación y la agricultura, Roma 2013. Disponible en: http://www.fao.org/3/a-i3437s.pdf

(17) Instituto de Hidrología, Meteorología y Estudios Ambientales IDEAM. Conceptos básicos de cambio climático. 2014. Disponible en: http://www.cambioclimatico.gov.co/otras-iniciativas

(18) Centro Internacional para la Investigación del Fenómeno de El Niño CIIFEN. Efecto invernadero. 2016. Disponible en: https:// goo.gl/G2ShQM

(19) Álvarez A. El cambio climático y la producción animal. Rev. Cubana de Ciencia Agrícola. 2014; 48(1): 7-10

(20) Organización de las Naciones Unidas para la Alimentación y la Agricultura FAO. El papel de la FAO en la producción animal. 2014. Disponible en: https://goo.gl/qdV5NF

(21) Hansen N., Fertig M., Tejera L. Componentes de los sistemas silvopastoriles en bosques de ñire. Rev. Forestal. 2009; 17: 77-82.

(22) Deambrosi A., Capozzolo M., Castro C. Sistemas silvopastoriles. Rev. Voces y Ecos. 29: 28-30.
(23) Instituto Interamericano de Cooperación para la Agricultura IICA. Establecimiento y uso de sistemas silvopastoriles en República Dominicana. 2016. Disponible en: https://goo.gl/SH1F6c

(24) Lamothe Y., León D., Moriche O. Cambio climático: silvopastoreo como alternativa para su mitigación. 2012. Disponible en: https://goo.gl/5azWcp

(25) Secretaría de Agricultura, Ganadería, Desarrollo Rural, Pesca y Alimentación SAGARPA. Sistemas Silvopastoriles. 2007. Disponible en: https://goo.gl/fY4uiO

(26) Mahecha L. El silvopastoreo: una alternativa de producción que disminuye el impacto ambiental de la ganadería bovina. Rev. Col Cienc Pec. 2002, 15(2): 226-231.

(27) Sadeghian S., Rivera J., Gómez M. Impacto de la ganadería sobre las características físicas, químicas y biológicas de suelos en los andes de Colombia. Agroforestería para la producción animal en América Latina. 1998, 123 -139.

(28) Zuluaga A., Zapata A., Uribe F., Murgueitio E., Cuartas C., Naranjo J., Molina C., Solarte L., Valencia L. Capacitación en establecimiento de sistemas silvopastoriles. Fondo Nacional del Ganado - Servicio Nacional de Aprendizaje FNG-SENA 2011. Disponible en: https://goo.gl/7GiCsF

(29) Belsky A.J., Mwonga S.M., Duxbury J.M. Efectos de los pastos ampliamente espaciados y el pastoreo de ganado en ambientes de sotobosque en sabanas tropicales. Rev. Sistemas Agroforestales. 1993, 24(1): 1-20. DOI: https://doi.org/10.1007/BF00705265.

(30) Fassbender H.W. Modelos edafológicos de sistemas agroforestales. Centro Agronómico Tropical de Investigación y Enseñanza CATIE, segunda Edición; 1993.

(31) Torres F. Papel de las perennes leñosas en la agroforestería animal. Rev. Sistemas Agroforestales. 1983, 1(2): 131-163. DOI: https://doi.org/10.1007/BF00596354.

(32) Santana M., Valencia J., Diaz C. Evaluación de tres sistemas silvopastoriles de Guayaba dulce (Psidium guajaba), Cañafistola (peltophorum dubium) y Guayaba - cañafistola, con Brachiaria humidicola en el bajo cauca antioqueño. Programa Nacional de Transferencia de Tecnología Agropecuaria PRONATTA. 1999. Disponible en: https://goo.gl/f1YnXm

(33) Petit J. Sistemas Silvopastoriles. Universidad de los Andes Venezuela - Instituto Nacional de Investigaciones Forestales, Agrícolas y Pecuarias Inifap 2012. Disponible en: https://goo.gl/zXs4Vx

(34) Putnam A. Alelopatía: problemas y oportunidades en el manejo de malezas. Organización de las Naciones Unidas para la Alimentación y la Agricultura FAO 1988. Disponible en: https:// goo.gl/Z2rioR

(35) Yandar A.R. Protocolos capacitación Actividad Silvopastoril. 2010. Disponible en: https://goo.gl/N7MgzS

(36) Alonso J. Los sistemas silvopastoriles y su contribución al medio ambiente. Rev. Cubana de Ciencia Agrícola. 2011; 45(2): 107115.

(37) Fernández Pascual M., María N., Rosario F. Fijación biológica del nitrógeno: factores limitantes. Ciencia y Medio Ambiente - Segundas jornadas científicas sobre medio ambiente del CCMA-CSIC. 2002 
(38) Centro Agronómico Tropical de Investigación y Enseñanza CATIE. Producción de madera en sistemas agroforestales de Centroamérica. 2012. Disponible en: https://goo.gl/jyUhpW

(39) Contextoganadero. Sistemas silvopastoriles con árboles frutales para alimentación del ganado. 2017. Disponible en: https://goo. gl/gZoXnU

(40) Veldkamp E. Dinámica del carbono orgánico del suelo en pastizales establecida después de la deforestación en los trópicos húmedos de Costa Rica. 1993. Disponible en: https://goo.gl/ $\mathrm{M} 4 \mathrm{mj} \mathrm{Ph}$

(41) Botero Botero J.A. Contribución de los sistemas ganaderos tropicales al secuestro de CO2. Organización de las Naciones Unidas para la Alimentación y la Agricultura FAO 2003. Disponible en: https://goo.gl/YYuXJR

(42) Castelvecchi D. Fotosíntesis. Rev. Investigación y Ciencia. 2009. Disponible en: https://goo.gl/EVix82

(43) Harvey C., Villanueva C., Villacıs J., Chacón M., Muñoz D., López M., Ibrahim M., Gómez R., Taylor R., Martínez J., Navas A., Sáenz D., Sánchez J., Medina A., Vílchez S., Hernández B., Pérez A., Ruiz F., López F., Lang I., Sinclair F. Contribución de las cercas vivas a la integridad ecológica de paisajes agrícolas. Rev. Agricultura, ecosistemas y medio ambiente. 2005; 111: 200-230. DOI: https://doi.org/10.1016/j.agee.2005.06.011.

(44) Wilson J-R. Crecimiento estimulado por la sombra y la absorción de nitrógeno por pastos en un ambiente subtropical. 1996. Disponible en: https://goo.gl/srEUtj.

(45) Casasola F., Ibrahim M., Sepulveda C., Rios N., Tobar D. Implementación de sistemas silvopastoriles y el pago de servicios ambientales en Esparza, Costa Rica: una herramienta para la adaptación al cambio climático en fincas ganaderas. Centro Agronómico Tropical de Investigación y Enseñanza (CATIE). 2009. Disponible en: https://goo.gl/1 RvbwV

(46) Beer J., Harvey C., Ibrahim M., Harmand J.M., Somarraba E., Jiménez F. Servicios ambientales de los sistemas agroforestales. Agroforestería en las Américas. 2003; 10: 80-87.

(47) Bolívar D., Ibrahim M., Jiménez O. Producción de brachiaria humidicola bajo un sistema silvopastoril con Acacia Mangium en el trópico húmedo. Centro Agronómico Tropical de Investigación y Enseñanza (CATIE). 1999. Disponible en: https://goo.gl/bpbroR

(48) Ibrahim M., Villanueva C.P., Casasola F. Sistemas silvopastoriles como una herramienta para el mejoramiento de la productividad y rehabilitación ecológica de paisajes ganaderos en centro américa. Arch. Latinoam. Prod. Anim. 2007; 15: 73-87.

(49) Blanco J. Una reseña sobre papel de la ganadería en los problemas actuales del medio ambiente. 2010. Disponible en: https:// goo.gl/8zFDyv

(50) Montagnini F. Sistemas silvopastoriles y mitigación del cambio climático: alternativas para aumentar la captura de C. Centro para la Investigación en Sistemas Sostenibles de Producción Agropecuaria CIPAV. 2010. Disponible en: https://goo.gl/yXm$1 \mathrm{Qc}$ 